\title{
«Wir sollten erst einmal die Tatsachen erfassen»
}

\author{
Interview: Bruno Kesseli
}

Dr. med. et lic. phil., Chefredaktor

\begin{abstract}
Die Genfer Ärztin und Bioethikerin Samia Hurst ist am Projekt «Entscheidungen am Lebensende: Häufigkeit und Trends in der Schweiz» beteiligt, das im Rahmen des Nationalen Forschungsprogramms 67 «Lebensende» läuft. In der Ärztekammer vom vergangenen Oktober hielt sie dazu ein vielbeachtetes Gastreferat. Das folgende Interview greift einige der damals angesprochenen Aspekte auf.
\end{abstract}

\begin{abstract}
Was ist unter dem Begriff "Entscheidungen am Lebensende» zu verstehen - können Sie Beispiele dazu geben?

Allgemein bezeichnen «Entscheidungen am Lebensende» sämtliche Entscheidungen, die am Lebensende getroffen werden, also nicht nur solche mit Bezug auf die Gesundheit. Im Rahmen der Forschung zu den Entscheidungen am Lebensende ist der Begriff jedoch präziser definiert. Er bezeichnet dann sämtliche Entscheidungen, die im Bewusstsein getroffen werden, dass sie die Lebensdauer verkürzen könnten, sei dies nun beabsichtigt oder nicht. Der Begriff bezeichnet somit auch alle medizinischen Entscheidungen, die in der Absicht getroffen werden, auf lebensverlängernde Massnahmen zu verzichten oder die Lebensdauer zu verkürzen.
\end{abstract}

\section{Warum ist es wichtig, über wissenschaftliche Daten zu Entscheidungen am Lebensende zu verfügen?}

Die Art und Weise, wie sich das Lebensende in der Schweiz gestaltet, liefert uns Hinweise auf bestimmte gesellschaftliche Entwicklungen. Unsere vergleichende Studie deutet beispielsweise auf einen Rückgang aggressiver lebensverlängernder Massnahmen

«Die Situation am Lebensende ist

darüber hinaus ein in Medizin und Gesellschaft kontrovers diskutiertes Thema.»

hin. Die Situation am Lebensende ist darüber hinaus ein in Medizin und Gesellschaft kontrovers diskutiertes Thema. Da ist es wichtig, dass die Argumente auf Fakten gründen. Allzu oft urteilen wir allein anhand unserer Vorstellungen darüber, ob diese oder jene Ent-

\section{Samia Hurst}

Prof. Dr. med. Samia Hurst ist Fachärztin für Innere Medizin, Bioethikerin und Direktorin des Instituts Ethique Histoire Humanité an der Universität Genf. Ausserdem ist sie Herausgeberin der Zeitschrift Bioethica Forum, Mitglied des Exekutivkomitees des Council for International Organizations of Medical Sciences (CIOMS) und der Nationalen Ethikkommission.

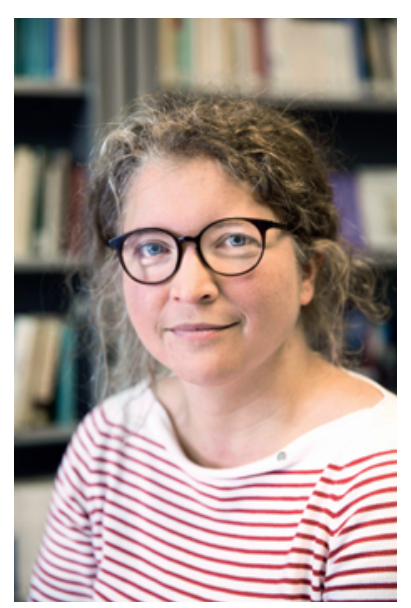

scheidung richtig oder falsch war. Dabei ist unser Vorstellungsvermögen sehr begrenzt. Wir sollten erst einmal die Tatsachen erfassen.

\section{Inwieweit hat die seit Jahren andauernde Debatte über die ärztliche Suizidhilfe die Lancierung des Forschungsprogramms beeinflusst?}

Diese Frage müsste man an primär an die Programmverantwortlichen richten. Nach meiner Einschätzung dürfte diese Debatte keine grössere Rolle gespielt haben. Es stimmt zwar, dass die Suizidhilfe in der Schweiz ein Dauerthema ist, allerdings ist das Forschungsfeld des NFP 67 viel weiter gefasst. Es beschäftigt sich mit den Sterbebedingungen und den gesellschaftlichen Vorstellungen vom Sterben in der Schweiz und geht damit weit über die Frage der Suizidhilfe hinaus, von der nur eine kleine Minderheit der Sterbenden in unserem Land betroffen ist. 


\section{Können Sie die wichtigsten Erkenntnisse der Studie kurz zusammenfassen?}

Derzeit geht der Hälfte der Todesfälle und 82\% der erwarteten und nicht plötzlich eingetretenen Todesfälle eine Entscheidung am Lebensende voraus. Die meisten dieser Entscheidungen betreffen den Behandlungsabbruch oder -verzicht und die Intensivierung der Symptombehandlung. Die Entwicklung in diesem Bereich ist nicht homogen. Während Behandlungsverzicht, palliative Sedierung und Suizidhilfe zugenommen haben, gilt dies nicht für die Intensivierung der Symptombehandlung, durch die das Sterben bewusst beschleunigt wird oder ein rascheres Sterben zumindest in Kauf genommen wird. Die Suizidhilfe, die am heftigsten debattiert wird, ist weiterhin sehr selten.

«Während Behandlungsverzicht, palliative Sedierung und Suizidhilfe zugenommen haben, gilt dies nicht für die Intensivierung der Symptombehandlung."

\section{Gab es Ergebnisse, von denen Sie und Ihre Forscher- kolleginnen und -kollegen überrascht waren?}

Wir haben untersucht, inwieweit Patientinnen und Patienten in Entscheidungen, die sie selbst betreffen, einbezogen werden. Es zeigte sich, dass die Einbeziehung der urteilsfähigen Patientinnen und Patienten abgenommen hat.

\section{Wie erklären Sie sich diesen Befund?}

Möglicherweise wird häufiger als früher davon ausgegangen, dass die Fortsetzung einer Behandlung nicht im Interesse des Patienten läge. In solchen Situationen können Ärztinnen und Ärzte zum Schluss kommen, dass sie dem Patienten keine Optionen vorschlagen müssen, die sie selbst für kontraindiziert halten. Dabei sollten sich solche Entscheidungen nach den Prioritäten des Patienten richten. Dieses Ergebnis scheint also problematisch.

\section{Lassen sich aus den Daten direkte praktische Konse- quenzen ableiten? Zum Beispiel für die Aus-, Weiter- und Fortbildung der Ärztinnen und Ärzte oder für deren praktische Tätigkeit?}

Entscheidungen am Lebensende haben zugenommen und müssen besser in die Aus- und vor allem Weiterbildung der Ärztinnen und Ärzte integriert werden. Der Stellenwert des Patienten bei solchen Entscheidungen muss immer wieder ins Gedächtnis gerufen und verdeutlicht werden. Auch wenn die Suizidhilfe weiterhin selten ist, wird die Tatsache, dass sie zunimmt, unweigerlich erneut die Frage nach ihrem Einbezug in die

\section{NFP 67 «Lebensende»}

Im Nationalen Forschungsprogramm «Lebensende» (NFP 67) untersuchen 33 Forscherteams aus unterschiedlichen disziplinären Perspektiven Aspekte des Lebensendes.

Das Ziel des NFP 67 besteht darin, Handlungs- und Orientierungswissen für den Bereich der letzten Lebensphase wissenschaftlich zu erarbeiten und dieses Entscheidungsträgerinnen und -trägern im Gesundheitswesen, in der Politik und den Berufsgruppen bereitzustellen, die sich mit der Betreuung von Menschen am Lebensende befassen.

Das NFP verfügt über einen Finanzrahmen von 15 Millionen CHF. Die Forschungsdauer beträgt 5 Jahre.

Weitere Informationen: www.nfp67.ch

medizinische Ausbildung aufwerfen. Dabei geht es nicht darum, Ärztinnen und Ärzte in der Suizidhilfe auszubilden, da niemand verpflichtet werden kann, diese zu leisten. Jedoch muss ein Arzt in der Lage sein, mit angemessenen Massnahmen zu reagieren, sollte ein Patient ihm gegenüber den Wunsch nach Suizidhilfe äussern. Diese Massnahmen könnten durchaus Gegenstand einer gezielteren Ausbildung sein.

\section{Wie werden die Ergebnisse nun weiter verwendet? Fliessen sie beispielsweise auch in politische Prozesse ein?}

Die Schweizerische Akademie der Medizinischen Wissenschaften überarbeitet derzeit ihre medizin-ethische Richtlinie zur Betreuung von Patientinnen und Patienten am Lebensende. Für die Arbeit der betreffenden Subkommission ist es sehr hilfreich, dass ihr ein Grossteil der Erkenntnisse aus dem NFP 67 zur Verfügung steht.

"Entscheidungen am Lebensende haben zugenommen und müssen besser in die Ausund vor allem Weiterbildung der Ärztinnen und Ärzte integriert werden."

Haben sich aus dem Programm neue Fragestellungen ergeben, die allenfalls in weiteren Studien untersucht werden sollten?

Die erhebliche Zunahme der palliativen Sedierung sollte in faktischer wie auch in ethischer Hinsicht näher untersucht werden.

Wie lautet Ihre persönliche Bilanz zum NFP «Entscheidungen am Lebensende»?

Das Projekt ist ein schönes Beispiel für die Möglichkeiten, die sich eröffnen, wenn die Forschung zu einem gesellschaftlich relevanten Thema mit öffentlichen Mitteln unterstützt wird. 